\title{
Applications of Conventional and A thermal Arrayed Waveguide Grating (AWG) Module in Active and Passive Optical Networks (PONs)
}

\author{
Abd El-Naser A. Mohammed and Ahmed Nabih Zaki Rashed
}

\begin{abstract}
In the present paper, we have investigated two characteristics of three different waveguides employed in arrayed waveguide grating (AWG) in passive optical networks (PON) where rates of variations are processed. Both the thermal and the spectral effects are taken into account. The waveguides are made of Lithium Niobate, germania-doped silica, and Polymethyl metha acrylate (PMMA) polymer. The thermal and spectral sensitivities of optical devices are also analyzed. In general, both qualitative and quantitative analysis of the temporal and spectral responses of AWG and sensitivity are parametrically processed over wide ranges of the set of affecting parameters.
\end{abstract}

Index Terms-Optical network, Arrayed waveguide gratings (AWGs), High integrated optics, planar waveguide, optical communications, Spectral and thermal sensitivities.

\section{INTRODUCTION}

The explosive growth of Internet traffic is pushing the rapid development of high-speed broadband optical networks, such as dense wavelength-division multiplexing (DWDM) systems. In these optical networks, a variety of optical components such as wavelength-division multi/demultiplexers (MUX/DEMUXs), erbium-doped fiber amplifiers (EDFAs), lasers, photodetectors [2-4], and modulators are indispensable for constructing optical networks [1]. Among others, arrayed waveguide grating (AWG)-type MUX/DEMUXs based on the optical planers circuits (PLCs) have played an important role as a key optical component for DWDM. Meanwhile, in the midst of the telecom winter, many carriers are struggling to reduce per-bit cost. Therefore [5-7], optical components are expected to have new functions that reduce the operational and capital expenditures. For example, an AWG itself is expected to be athermal as well as low cost. In order to lower the cost of AWGs, besides testing and packaging costs, chip size is quite important because the number of AWGs laid out over a wafer determines the general cost. This is especially true for large-size optical circuits such as AWGs. The increase in refractive index difference between core and cladding is a

Ahmed Nabih Zaki Rashed is with Electronics and Electrical Communication Engineering Department, faculty of Electronic Engineering, Menouf 32951, Menoufia University, EGYPT. Corresponding author to provide phone. [Tel.: +2 048-3660-617, Fax: +2 048-3660-617]. quite useful way to reduce chip size. So far, several types of high-contrast waveguides have been reported to have been achieved by using several material systems, such as a semiconductor waveguide[8], SiON waveguide [9], Si-wire waveguides [10], and silica-based waveguide [11]. Among these waveguides, the silica-based waveguide is suitable in the light of low propagation loss as well as high reliability.

The periodic and cyclic properties of AWGs are used to interconnect two adjacent ONUs by a piece of fiber. In the recent years, arrayed waveguide gratings (AWGs) have appeared to be one of attractive candidates for high channel count Mux/DeMux devices to process optical signals in a parallel manner. Its low chromatic dispersion, typically \pm 5 $\mathrm{ps} / \mathrm{nm}- \pm 10 \mathrm{ps} / \mathrm{nm}$, makes it possibly be used for $40 \mathrm{Gbit} / \mathrm{s}$ systems. However, it is well known that manufacturing AWGs involves a series of complex production processes and requires bulky facilities. Their cost remains a big issue. Further, the technical complexity leads to low yield and poor performance. The former, no doubt, further increases the production cost while the latter degrades the signal quality and system's performance, exhibiting high insertion loss, high channel crosstalk, low channel uniformity, and high polarization dependent loss. More vitally, AWGs require active temperature control in order to stabilize the thermal wavelength drift and temperature. Due to its capability to increase the aggregate transmission capacity of a single-strand optical fiber, the arrayed waveguide grating (AWG) multiplexer is considered a key component in the construction. material and PMMA cladding material is considered as the most attractive a thermal structure because of its resistance to the thermo-optic sensitivity of the materials. Arrayed Waveguide Gratings (AWGs) have increasingly become more important in Wavelength Division Multiplexing (WDM) systems. An AWG in silicon-on-insulator (SOI) is of particular interest because of both its technological andmaterial advantages [12]. The high index contrast in the SOI optical system results in a smaller device and better optical confinement within the waveguide as compared to low index contrast systems [13]. Also, the mature microelectronic fabrication technology can be easily transferred to most of the SOI photonic devices. The spectral response of a typical SOI AWG exhibits a Gaussian profile, which restricts the wavelength selectivity of the devices due to wavelength drift caused by the laser diode and the AWG. Therefore [14], a flat spectral response is desirable to ease the wavelength selectivity of the WDM system. Various methods 
have been proposed and demonstrated in different material systems and each has its own advantages and disadvantages [15]. To date, with the explosive growth of end user demand for higher bandwidth, various types of passive optical networks (PONs) have been proposed. PON can be roughly divided into two categories such as time-division-multiplexing (TDM) and wavelength-division-multiplexing (WDM) methods [16]. Compared with TDM-PONs, WDM-PON systems allocate a separate wavelength to each subscriber, enabling the delivery of dedicated bandwidth per optical network unit (ONU). Moreover, this virtual point-to-point connection enables a large guaranteed bandwidth, protocol transparency, high quality of service, excellent security, bit-rate independence, and easy upgradeability. Especially, recent good progress on athermal arrayed waveguide grating (AWG) and cost-effective colorless ONUs [17] has empowered WDM-PON as an optimum solution for the modern optical access telecommunication networks.

Arrayed waveguide grating (AWG) which handles the function of wavelength multiplexer/demultiplexer is extensively used in configuring optical communication networks that are becoming more diversified. Since the transmission wavelength of an AWG is temperature dependent, it was a common practice to control its temperature using heaters or Peltier elements. But this caused problems of power consumption increase in the whole system in addition to limiting the installation locations of AWGs, thus making it difficult to respond to various needs of the next-generation optical communication networks, despite the fact that system performance upgrading and function enhancement are required in recent years. However, fiber link failure from the optical line terminal (OLT) to the ONU leads to the enormous loss of data. Thus, fault monitoring [18] and network protection [19] are crucial issues in network operators for reliable network. To date, many methods have been proposed for network protection. In the ITU-T recommendation on PONs (G.983.1), duplicated network resources such as fiber links or ONUs are required. Planar light-wave circuit technology and design have evolved significantly in the past decade, in terms of both performance and yield. New semiconductor techniques applied to integrated optics have dramatically improved wafer quality; in parallel, design efforts have led to lowering insertion loss, reducing crosstalk, increasing channel bandwidth, decreasing channel spacing, and managing chromatic dispersion (CD). With arrayed waveguide gratings (AWGs) that match or exceed the performance of thin-film filters, and will enable with the integration of variable optical attenuators and monitoring taps to realize high-performance and low-cost modules with added functions for the system. Desirable characteristics of any AWG device include low loss in the passbands, high loss outside the passbands, uniform loss within one channel and channel-to-channel, and polarization-independent behavior. While low crosstalk is of paramount importance in demultiplexers where out-of-band signals appear as noise at the receiver, it is of little concern in multiplexers where out-of-band signals simply are not present in the transmitter. For multiplexing, a flat response within the passband is highly desirable in order to account for wavelength drift in the laser source. The explosion in demand for Internet services is promoting the rapid development of high-speed and broad-and optical networks such as fiber to the home (FTTH) and dense wavelength division multiplexing (DWDM) systems. Such networks require a variety of optical components including micro optics, fiber, and planar-type elements. Planar light wave circuits (PLC) fabricated using silica-based waveguides are employed in various devices because of their excellent design flexibility, stability, and mass-reducibility. Of the PLC-type devices, arrayed waveguide gratings (AWGs) are superior to other types of wavelength multi/demultiplexers, such as dielectric multilayered filters, in terms of compactness and multichanneling. Today, optical networks are required to be more broad-band and economical. Therefore, it is hoped that DWDM systems will increase their capacity and spread throughout the access networks. At the same time, there is also a need for AWG multi/demultiplexers to be further improved in terms of channel count and made more compact and less expensive. Moreover, the functionsand scale of PLCs should be enhanced to allow us to construct future photonic networks that need advanced optical processing such as optical switching and routing. To meet these requirements, it is important that we improve PLCs in terms of low-loss and high-density integration [20].

In the present study, both the thermal and spectral variations of three waveguides made of different materials are deeply and parametrically investigated over wide range of the affecting parameters. Thermal sensitivity and spectral sensitivity are also of major interest in photonic integrated circuits (PIC).

\section{SYSTEM MODEL ANALYSIS}

\section{A. LITHIUM NIOBATE (LiNbO3) MATERIAL}

The investigation of both the thermal and spectral variations of the waveguide refractive index (n) require Sellmeier equation. The set of parameters required to completely characterize the temperature dependence of the refractive-index (n) is given below, Sellmeier equation is under the form [21]:

$$
n^{2}=A_{1}+A_{2} H+\frac{A_{3}+A_{4} H}{\lambda^{2}-\left(A_{5}+A_{6} H\right)^{2}}+\frac{A_{7}+A_{8} H}{\lambda^{2}-A_{9}^{2}}-A_{10} \lambda^{2}
$$

where $\lambda$ is the optical

wavelength in $\mu \mathrm{m}$ and $H=T^{2}-T_{0}^{2}$. T is the temperature of the material, $\mathrm{K}$, and $\mathrm{T}_{0}$ is the reference temperature and is considered as $300 \mathrm{~K}$. The set of parameters of Sellmeier equation coefficients $\left(\mathrm{LiNbO}_{3}\right)$ are recast and dimensionally adjusted as below [21]: $\mathrm{A}_{1}=5.35583, \mathrm{~A}_{2}=4.629 \times 10^{-7}$, $\mathrm{A}_{3}=0.100473, \mathrm{~A}_{4}=3.862 \times 10^{-8}, \mathrm{~A}_{5}=0.20692, \mathrm{~A}_{6}=-0.89 \times 10^{-8}$, $\mathrm{A}_{7}=100, \mathrm{~A}_{8}=2.657 \times 10^{-5}, \mathrm{~A}_{9}=11.34927$, and $\mathrm{A}_{10}=0.01533$. Equation (1) can be simplified as:

$$
n^{2}=A_{12}+\frac{A_{34}}{\lambda^{2}-A_{56}^{2}}+\frac{A_{78}}{\lambda^{2}-A_{9}^{2}}-A_{10} \lambda^{2}
$$

where: $\mathrm{A}_{12}=\mathrm{A}_{1}+\mathrm{A}_{2} \mathrm{H}, \mathrm{A}_{34}=\mathrm{A}_{3}+\mathrm{A}_{4} \mathrm{H}, \mathrm{A}_{56}=\mathrm{A}_{5}+\mathrm{A}_{6} \mathrm{H}$, and $\mathrm{A}_{78}=\mathrm{A}_{7}+\mathrm{A}_{8} \mathrm{H}$.

Then, the differentiation of Eq. (2) w. r. $t \lambda$ gives: 


$$
\frac{d n}{d \lambda}=\left(\frac{-\lambda}{n}\right)\left[\frac{A_{34}}{\left(\lambda^{2}-A_{56}^{2}\right)^{2}}+\frac{A_{78}}{\left(\lambda^{2}-A_{9}^{2}\right)^{2}}+A_{10}\right]
$$

Also, the differentiation of Eq. (2) w. r. $\mathrm{t}$ T gives:

$$
\frac{d n}{d T}=\left(\frac{T}{n}\right)\left[A_{2}+\frac{\left(\lambda^{2}-A_{56}^{2}\right) A_{4}+2 A_{6} A_{56} A_{34}}{\left(\lambda^{2}-A_{56}^{2}\right)^{2}}+\frac{A_{8}}{\left(\lambda^{2}-A_{9}^{2}\right)}\right]
$$

\section{B. GERMANIA DOPED SILICA (GeO2(x)+SiO2(1-x)) MATERIAL}

The refractive index of this waveguide is cast as [22]:

$$
n^{2}=1+\frac{B_{1} \lambda^{2}}{\lambda^{2}-B_{2}^{2}}+\frac{B_{3} \lambda^{2}}{\lambda^{2}-B_{4}^{2}}+\frac{B_{5} \lambda^{2}}{\lambda^{2}-B_{6}^{2}}
$$

The Sellemier coefficients as a function of temperature, and germania mole fraction, $\mathrm{x}$, as follows:

$$
\begin{aligned}
\mathrm{B}_{1} & =0.691663+0.1107001 * \mathrm{x}, \\
\mathrm{B}_{2} & =(0.0684043+0.000568306 * \mathrm{x})^{2} *\left(\mathrm{~T} / \mathrm{T}_{0}\right)^{2}, \\
\mathrm{~B}_{3} & =0.4079426+0.31021588 * \mathrm{x}, \\
\mathrm{B}_{4} & =(0.1162414+0.03772465 * \mathrm{x})^{2} *\left(\mathrm{~T} / \mathrm{T}_{0}\right)^{2}, \\
\mathrm{~B}_{5} & =0.8974749-0.043311091 * \mathrm{x}, \\
\text { and } \mathrm{B}_{6} & =(9.896161+1.94577 * \mathrm{x})^{2} .
\end{aligned}
$$

The differentiation of Eq. (5) w. r. $\mathrm{t} \lambda$ gives:

$$
\begin{aligned}
& \frac{d n}{d \lambda}=-(\lambda / n) \\
& {\left[\frac{B_{1} B_{2}^{2}}{\left(\lambda^{2}-B_{2}^{2}\right)^{2}}+\frac{B_{3} B_{4}^{2}}{\left(\lambda^{2}-B_{4}^{2}\right)^{2}}+\frac{B_{5} B_{6}^{2}}{\left(\lambda^{2}-B_{6}^{2}\right)^{2}}\right]}
\end{aligned}
$$

Also, the differentiation of Eq. (5) w. r. t T yields:

$$
\frac{d n}{d T}=\left(-\lambda^{2} / n\right)\left[\frac{B_{1} B_{2} \frac{\partial B_{2}}{\partial T}}{\left(\lambda^{2}-B_{2}^{2}\right)^{2}}+\frac{B_{3} B_{4} \frac{\partial B_{4}}{\partial T}}{\left(\lambda^{2}-B_{4}^{2}\right)^{2}}+\frac{B_{5} B_{6} \frac{\partial B_{6}}{\partial T}}{\left(\lambda^{2}-B_{6}^{2}\right)^{2}}\right]
$$

\section{POLYMETHYL-METHA ACRYLATE (PMMA) POLYMER MATERIAL}

The refractive index of this waveguide is cast as [23]:

$$
n^{2}=1+\frac{C_{1} \lambda^{2}}{\lambda^{2}-C_{2}^{2}}+\frac{C_{3} \lambda^{2}}{\lambda^{2}-C_{4}^{2}}+\frac{C_{5} \lambda^{2}}{\lambda^{2}-C_{6}^{2}}
$$

The set of parameters of Sellmeier equation coefficients (PMMA) are recast below [23]:

$\mathrm{C}_{1}=0.4963, \mathrm{C}_{2}=0.0718\left(\mathrm{~T} / \mathrm{T}_{0}\right), \mathrm{C}_{3}=0.6965$,

$\mathrm{C}_{4}=0.1174\left(\mathrm{~T} / \mathrm{T}_{0}\right), \mathrm{C}_{5}=0.3223$, and $\mathrm{C}_{6}=9.237$. where $\mathrm{T}$ is the temperature of the material, and $\mathrm{T}_{0}$ is the reference temperature. The differentiation of Eq. (8) w. r. $\mathrm{t} \lambda$ gives:

$$
\begin{aligned}
& \frac{d n}{d \lambda}=-(\lambda / n) \\
& {\left[\frac{C_{1} C_{2}^{2}}{\left(\lambda^{2}-C_{2}^{2}\right)^{2}}+\frac{C_{3} C_{4}^{2}}{\left(\lambda^{2}-C_{4}^{2}\right)^{2}}+\frac{C_{5} C_{6}^{2}}{\left(\lambda^{2}-C_{6}^{2}\right)^{2}}\right]}
\end{aligned}
$$

Also, the differentiation of Eq. (8) w. r. t T yields:

$$
\frac{d n}{d T}=\left(-\lambda^{2} / n\right)\left[\frac{C_{1} C_{2} \frac{\partial C_{2}}{\partial T}}{\left(\lambda^{2}-C_{2}^{2}\right)^{2}}+\frac{C_{3} C_{4} \frac{\partial C_{4}}{\partial T}}{\left(\lambda^{2}-C_{4}^{2}\right)^{2}}+\frac{C_{5} C_{6} \frac{\partial C_{6}}{\partial T}}{\left(\lambda^{2}-C_{6}^{2}\right)^{2}}\right]
$$

\section{SENSITIVITIES OF WAVEGUIDES}

In fact, the thermal sensitivity $S_{T}^{n}$ of $\mathrm{n} \mathrm{w}$. r. $\mathrm{t} T$ is defined as follows:

$$
S_{T}^{n}=\left(\frac{T}{n}\right) \frac{d n}{d T}
$$

and the spectral sensitivity $S_{\lambda}^{n}$ of $\mathrm{n} \mathrm{w}$. r. $\mathrm{t} \lambda$ is defined as follows:

$$
S_{\lambda}^{n}=\left(\frac{\lambda}{n}\right) \frac{d n}{d \lambda}
$$

\section{RESULTS AND DISCUSSIONS}

Thermal and spectral variations of $\mathrm{n}$ for the three waveguides are displayed in Figs. (1-6), these figures assure the following:

1- $(\mathrm{dn} / \mathrm{dT}$ or $\mathrm{dn} / \mathrm{d} \lambda)$ against the variations of $(\mathrm{T}$ or $\lambda)$ at constant $(\lambda$ or $\mathrm{T})$ possesses either positive or negative correlations for three optical waveguides.

2- As the wavelength increases, $(\mathrm{dn} / \mathrm{dT}$ or $\mathrm{dn} / \mathrm{d} \lambda)$ of $\mathrm{LiNbO}_{3}$ material decrease at constant $\mathrm{T}$, and as the temperature increases, $(\mathrm{dn} / \mathrm{dT}$ or $\mathrm{dn} / \mathrm{d} \lambda)$ of $\mathrm{LiNbO}_{3}$ material increase at constant $\lambda$. This indicates its thermal stability of $\mathrm{LiNbO}_{3}$ material, $\mathrm{dn} / \mathrm{dT}$ is constant at (T or $\lambda$ ).

3- As the wavelength increases, $(\mathrm{dn} / \mathrm{dT}$ or $\mathrm{dn} / \mathrm{d} \lambda)$ of Silica-doped material increase at constant $\mathrm{T}$, and as the temperature increases, $(\mathrm{dn} / \mathrm{dT}$ or $\mathrm{dn} / \mathrm{d} \lambda)$ of Silica-doped material also increase at constant $\lambda$. This indicates its spectral stability of Silica-doped material, $\mathrm{dn} / \mathrm{d} \lambda$ is constant at ( $\mathrm{T}$ or $\lambda)$.

4- As the wavelength increases, $(\mathrm{dn} / \mathrm{dT}$ or $\mathrm{dn} / \mathrm{d} \lambda)$ of PMMA Polymer material decrease at constant $\mathrm{T}$, and as the temperature increases, $(\mathrm{dn} / \mathrm{dT}$ or $\mathrm{dn} / \mathrm{d} \lambda)$ of PMMA Polymer material also decrease at constant $\lambda$.

Thermal and spectral variations of $S_{T}^{n}$ and $S_{\lambda}^{n}$ for the three waveguides are also displayed in Figs. (7-12), the following features can be concluded:

5 As the wavelength increases, both the thermal and spectral sensitivities of LiNbO3 material decrease at constant $\mathrm{T}$, and as the temperature increases, the thermal sensitivity of LiNbO3 material increase at constant $\lambda$.

6- As the wavelength increases, both the thermal and spectral sensitivities of Silica-doped material increase at constant $\mathrm{T}$, and as the temperature increases, also both the thermal and spectral sensitivities of Silica-doped material increase at constant $\lambda$.

7- As the wavelength increases, both the thermal and spectral sensitivities of PMMA Polymer material7- As the wavelength increases, both the thermal and spectral sensitivities of PMMA Polymer material decrease at constant 
$\mathrm{T}$, and as the temperature increases, the thermal sensitivity of PMMA Polymer material increase at constant $\lambda$.

\section{CONCLUSIONS}

In a summary, three passive optical waveguides employed in PON and made of either Lithium niobate, Silica-doped fiber, and PMMA polymer fiber are spectrally and thermally investigated based on Sellmeier equation. Thermal and spectral sensitivities are also investigated. Positive correlations or negative correlations or both are found. In general, the three waveguides possess weak nonlinear correlations.

\section{REFERENCES}

[1] Y. Hibino. Recent Advances in High Density and Large Scale AWG Multi/Demultiplexers With Higher Index-Contrast Silica -Based PLCs IEEE J. Selected Topics Quantum Elect, vol. 8, no. 3, pp. 1090-1101, 2000.

[2] M. C. Parker, and S. D. Walker. Design of Arrayed Waveguide Grating Using Hybrid Fourier Fresnel Transform Techniques. IEEE J. Selected Topics Quantum Elect, vol. 5, no. 2, 1379-1384, 1999.

[3] P. Munoz, D. Pastor and J. Capmany. Analysis and Design of Arrayed Waveguide Gratings with MMI Couplers. Optics Express, vol. 9, no. 5, pp. 328-330, 2000.

[4] P. Munoz, D. Pastor and J. Capmany. Modelling and Design of Arrayed Waveguide Gratings. J. of Lightwave Technol., vol. 20, no. 2, pp. 661-674, 2000 .

[5] K. Noguchi, Y. Koike, H. Tanobe, K. Harada, and M. Matsuoka. Field Trial of Full-Mesh WDM Network (AWG-STAR) in Metropolitan/Local Area. J. of Lightwave Technol., vol. 22, no. 3, pp. 329-337, 2002

[6] Hu, Sai. M.S.C.Ersoy. Design and Simulation of Novel Arrayed Waveguide Grating by Using the Method of Irregularly Sampled Zero Crossings. IEEE Quantum Electronics., vol. 35, no. 6, pp. 1233-1249, 2002.

[7] E-S. Kang, W-S. Kim, D-J. Kim, and B-S. Bae. Reducing the Thermal Dependence of Silica-Based Arrayed Waveguide Grating Using Inorganic-Organic Hybrid Materials. IEEE Photonics Technol. Letters, vol. 16, no. 1, pp. 2625-2627, 2003.

[8] B. Fondeur, A. L. Sala, H. Yamada, R. Brainard, E. Egan, S. Thekdi, N. Gopinathan, D. Nakamoto, and A. Vaidyanathan. Ultrawide AWG With Hyper-Gaussian Profile. IEEE Photonics Technol. Letters, vol. 16, no. 3, pp. 2628-2630, 2004.

[9] A-A. Saleh and J. M. Simmons. Evolution Toward the Next Generation Core Optical Network. J. of Lightwave Technol., vol. 24, no. 2, pp. 3303-3320, 2006.

[10] K. Suzuki, Y. Hida, T. Shibata, Y. Inone, H. Takahashi, and K. Okamoto. Silica-Based Arrayed Waveguide Gratings For the Visible Wavelength Range. NTT Techanical Review, vol. 4, no. 1, pp. 48-51, 2002.

[11] O. M. Matos, M. L. Calvo, P. Cheben, S. Janz, J. A. Rodrigo, D-X. Xu, and A. Delage. Arrayed Waveguide Grating Based on Group Index Modification. J. of Lightwave Technol., vol. 24, no. 5, pp. 1551-1559, 2006.

[12] K. Maru, Y. Abe, M. Ito, H. Ishikaura, S. Himi, H. Uetsuka, and T. Mizumoto. $2.5 \%-\Delta$ Silica-Based A thermal Arrayed Waveguide Grating Employing Spot Size Converter Based on Segmented Core. IEEE Photonics Technol. Letters, vol. 17, no. 2, pp. 2325-2327, 2005.
[13] Photeon Technologies. The Arrayed Waveguide Gratings, pp. 1-5, 2005. www.photeon. Com.

[14] H. Uetsuka. AWG Technologies for Dense WDM Applications. IEEE Quantum Electronics, vol. 10, no. 2, pp. 393-402, 2005.

[15] L. G. Deperalta, A. A. Bernussi, V. Gorbounor, and H. Temakin. Temperature Insensitive Refractive Arrayed Waveguide Grating Multiplexers. IEEE Photonics Technol. Letters, vol. 16, no. 6, pp. 831-833, 2005.

[16] M. K. Smit. Progress in AWG Design and Technology. IEEE Quantum Electronics, vol. 18, no. 2, pp. 1-5, 2004.

[17] A. Gholipour, and R. F. Dana. NonUniform Arrayed Waveguide Gratings For Flat Top Passband Transfer Function. J. of Lightwave Technol., vol. 25, no. 1, pp. 3678-3683, 2005.

[18] H. C. Lu, and W. S. Wang. Cyclic Arrayed Waveguide Grating Devices With Flat Top Passband and Uniform Spectral Response. IEEE Photonics Technol. Letters, vol. 20, no. 3, pp. 1-5, 2003.

[19] D. Robertson, P. Niewczas, J. R. McDonald. Interrogation of a Dual Fiber Bragg Grating Sensor Using an Arrayed Waveguide Grating. IEEE Transaction On Instrumentation and Measurements, vol. 56, no. 9, pp. 2641-2645, 2007.

[20] M. Simmons. Survivable Passive Optical Networks Based on Arrayed Waveguide Grating Architectures. J. of Lightwave Technol., vol. 25, no. 3, pp. 3658-3669, 2005.

[21] D. H. Jundt. Fabrication Techniques of Lithium Niobate Waveguides. Optics Letters, vol. 22, no. 1, pp. 1553-1555, 1997.

[22] W. Fleming. Dispersion in $\mathrm{GeO}_{2}-\mathrm{SiO}_{2}$ Glasses. Applied Optics, vol. 23, no. 24, pp. 4486-4493, 1985.

[23] T. Ishigure, E. Nihei, and Y. Koike. Optimum Refractive Index Profile of The Grade-Index Polymer Optical Fiber Toward Gigabit Data Link. Appl. Opt., vol. 35, no. 22, pp. 2048-2053, 1996.

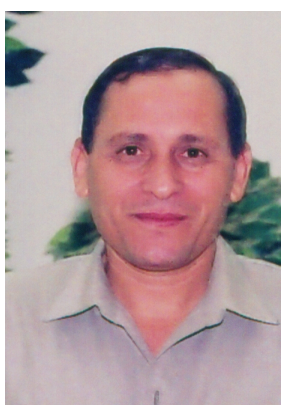

Abd-EInaser A. Mohammed

Received Ph.D degree from the faculty of Electronic Engineering, Menoufia University in 1994. Now, his job career is Assoc. Prof. Dr. in Electronics and Electrical Communication Engineering department. Currently, his field and research interest in the passive optical communication Networks,

analog digital communication systems, and advanced optical communication networks.

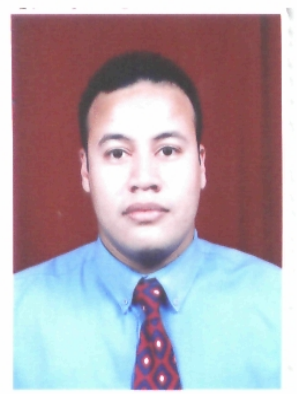

\section{Ahmed Nabih Zaki Rashed}

was born in Menouf, Menoufia State, Egypt, in 1976. Received the practical B.Sc. and M.Sc. scientific practical degrees in the Electronics and Electrical Communication Engineering Department from Faculty of Electronic Engineering, Menoufia University in 1999 and 2005, respectively. Currently, his field interest and working toward the Ph.D degree in Active and Passive Optical Networks (PONs). His research mainly focuses on the transmission data rate of optical networks. 


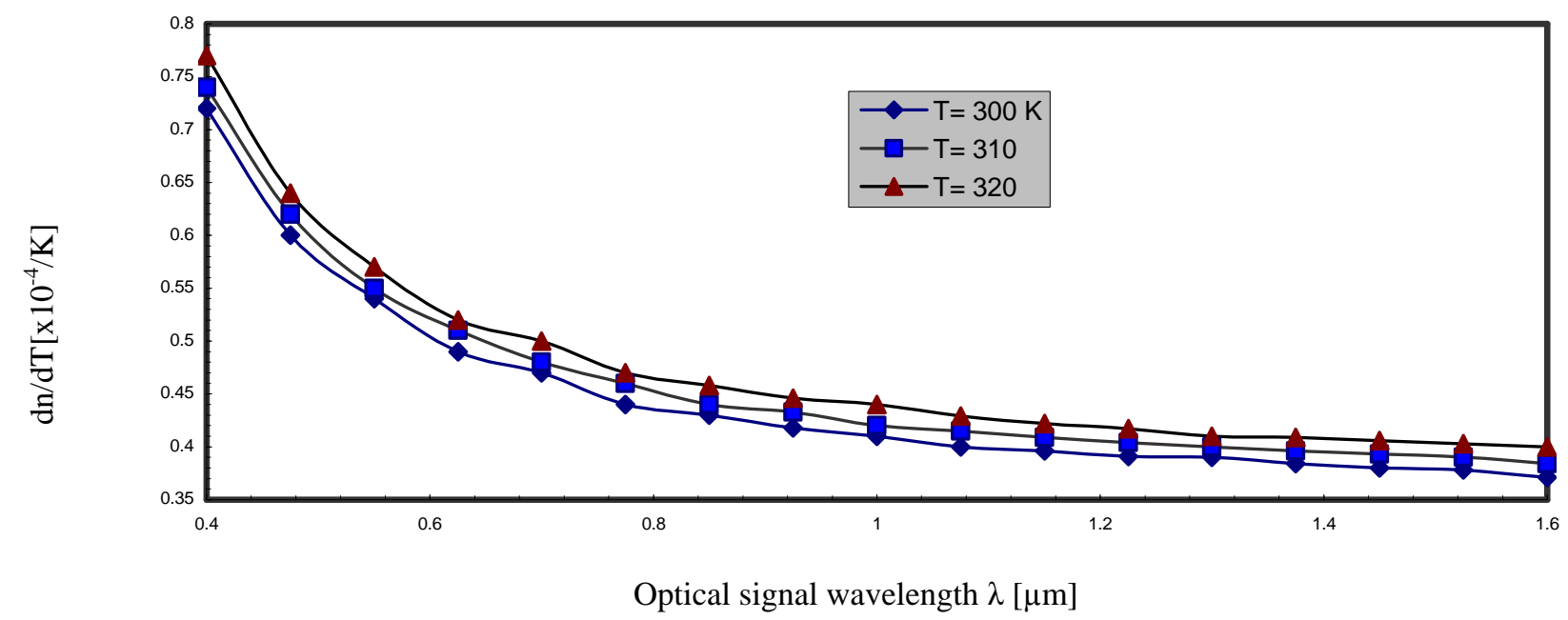

Fig. 1. Variation of dn/dT versus wavelength for $\mathrm{LiNbO}_{3}$ material

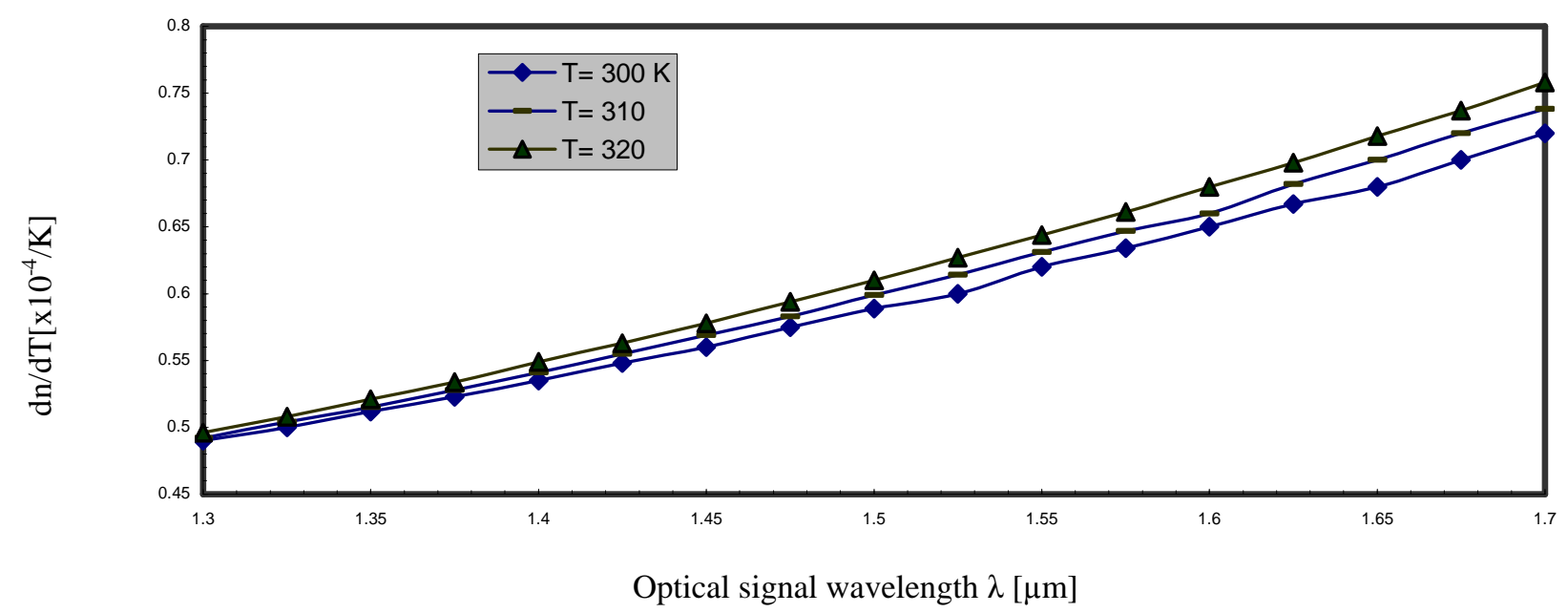

Fig. 2. Variation of dn/dT versus wavelength for Silica- doped material

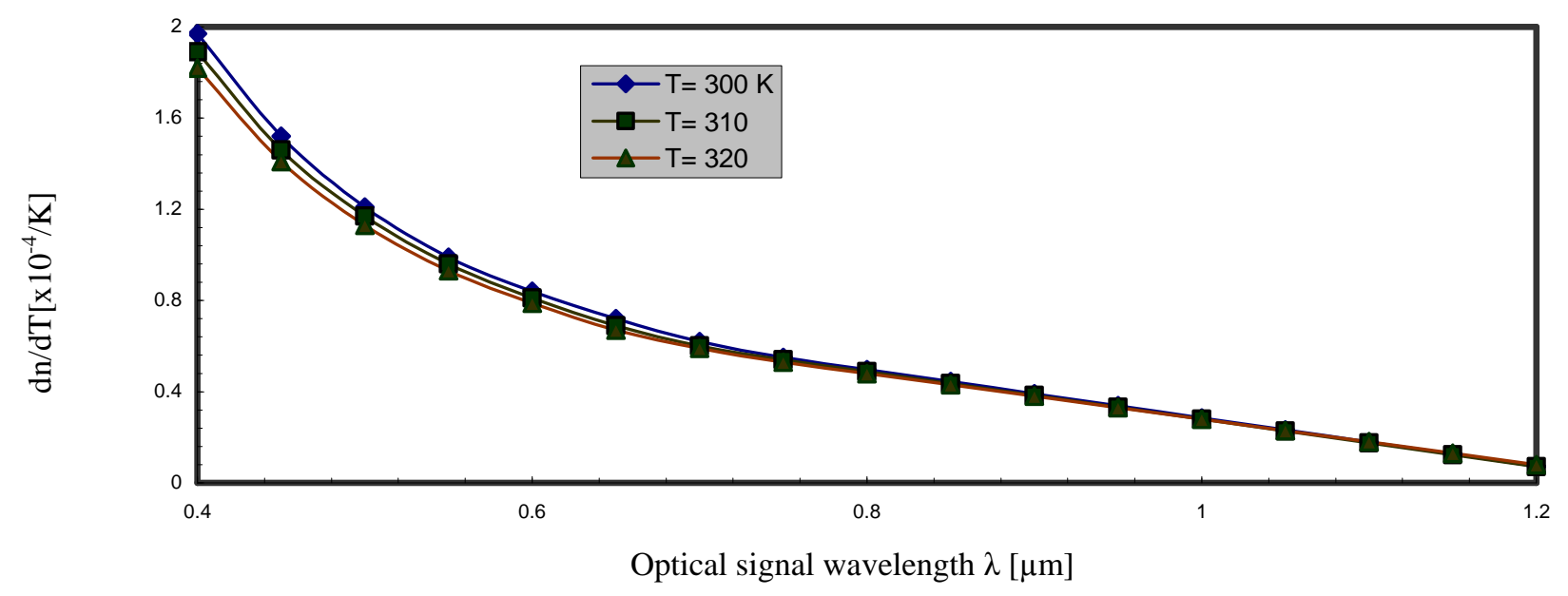

Fig. 3. Variation of dn/dT versus wavelength for PMMA material 


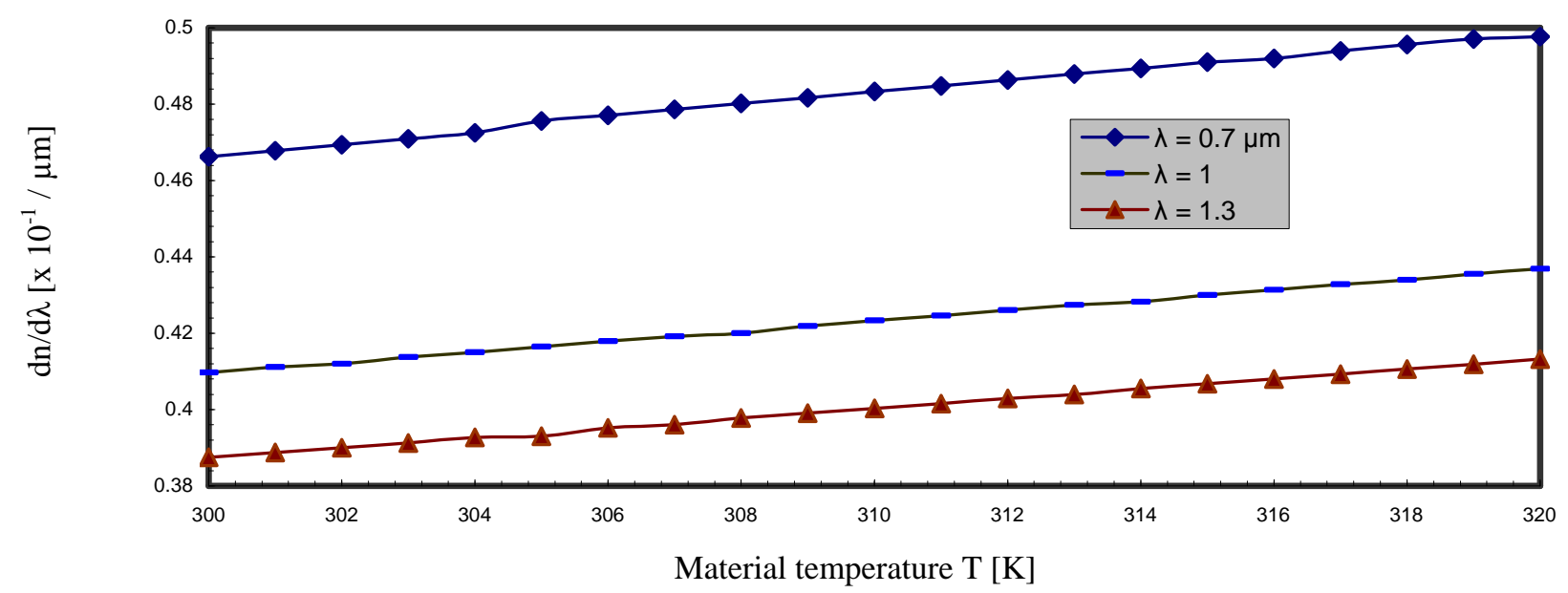

Fig. 4. Variation of $\mathrm{dn} / \mathrm{d} \lambda$ versus temperature for $\mathrm{LiNbO}_{3}$ material

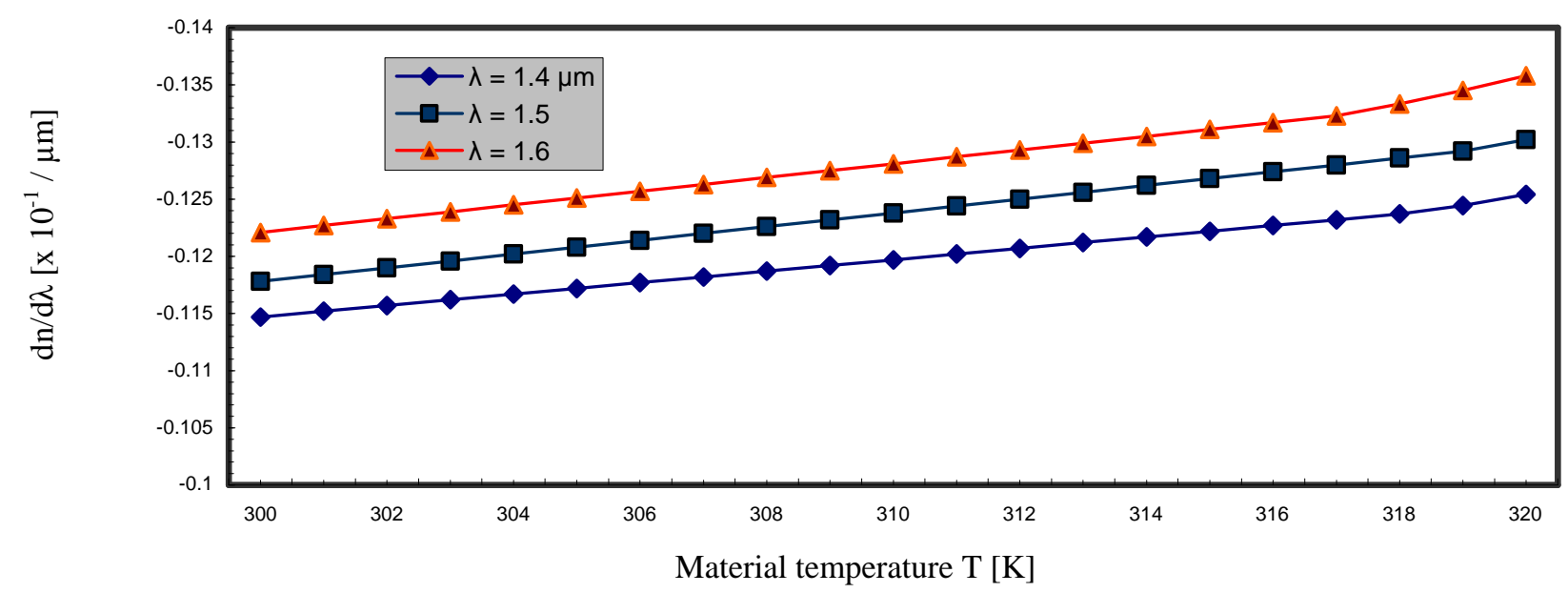

Fig. 5. Variation of $\mathrm{dn} / \mathrm{d} \lambda$ versus temperature for Silica-doped material

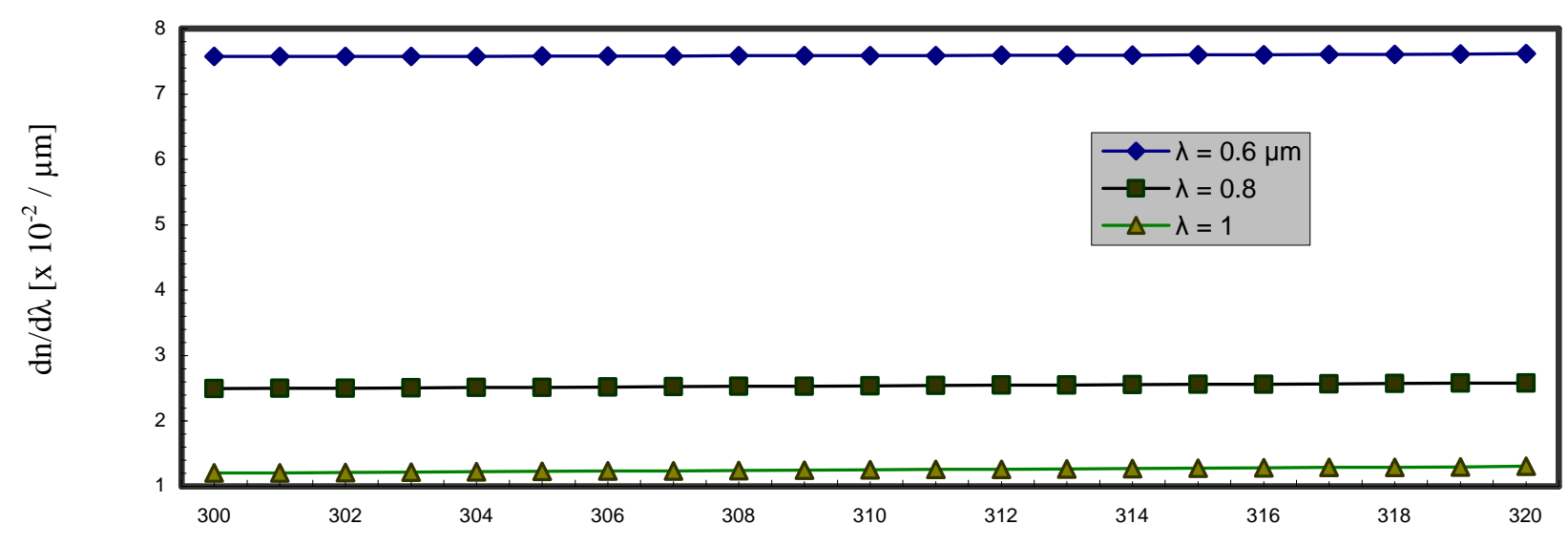

Material temperature $\mathrm{T}[\mathrm{K}]$

Fig. 6. Variation of $\mathrm{dn} / \mathrm{d} \lambda$ versus temperature for PMMA material 


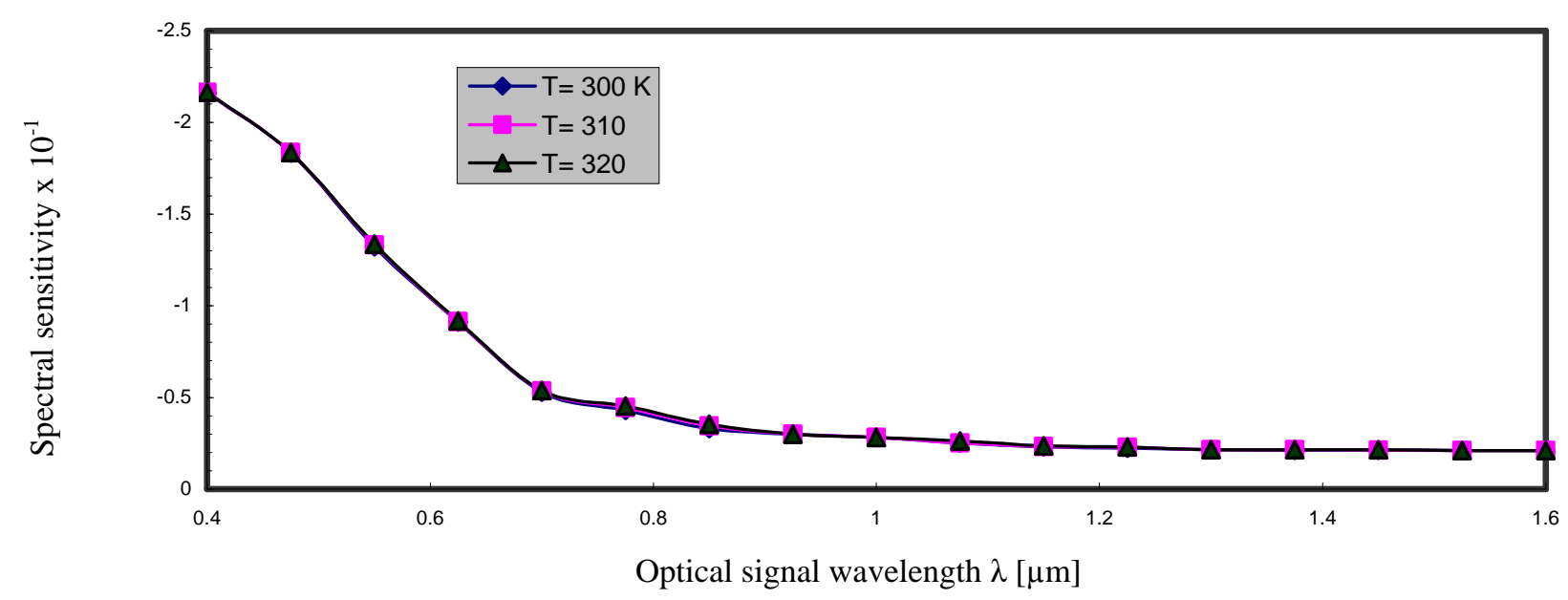

Fig. 7. Variation of spectral sensitivity versus wavelength for $\mathrm{LiNbO}_{3}$ material

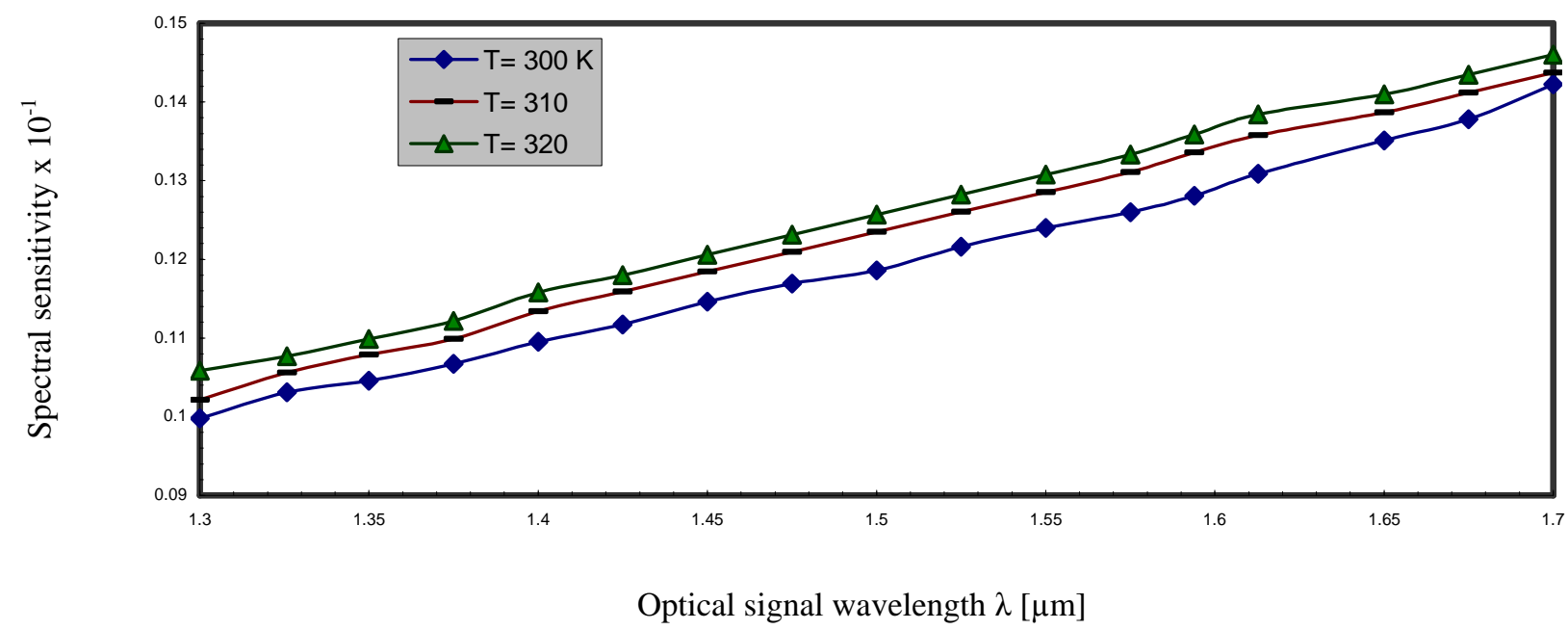

Fig. 8. Variation of spectral sensitivity versus wavelength for Silica-doped material

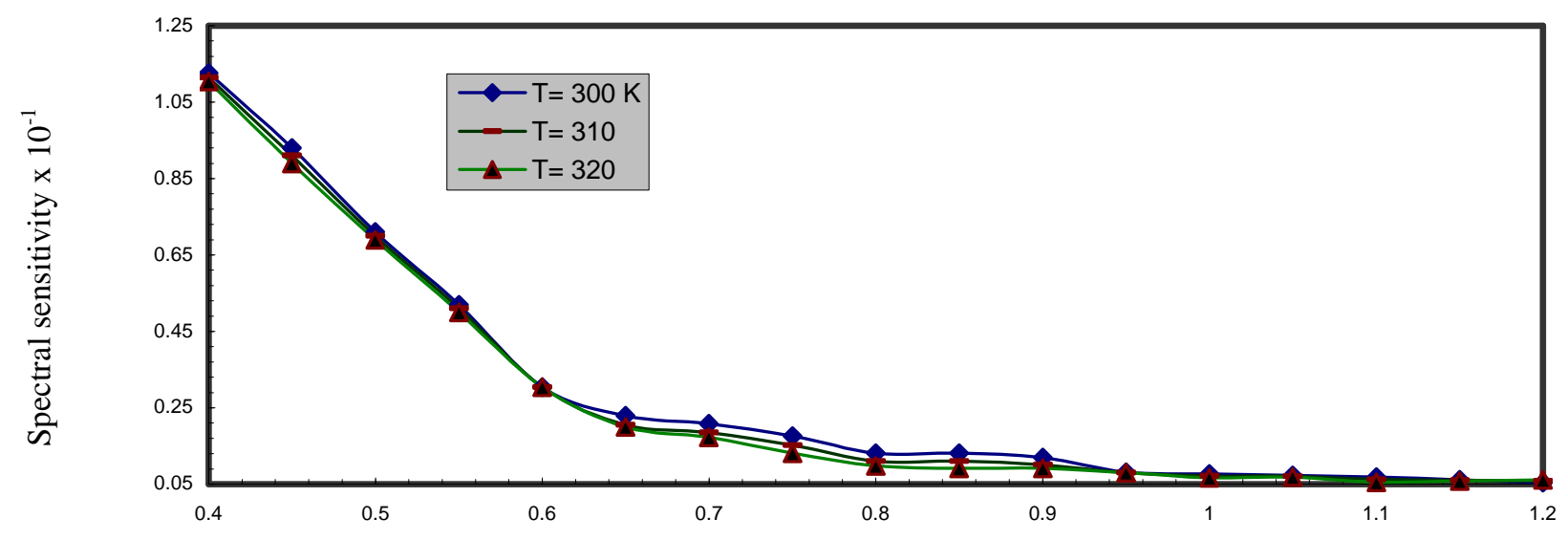

Optical signal wavelength $\lambda[\mu \mathrm{m}]$

Fig. 9. Variation of spectral sensitivity versus wavelength for PMMA materia 


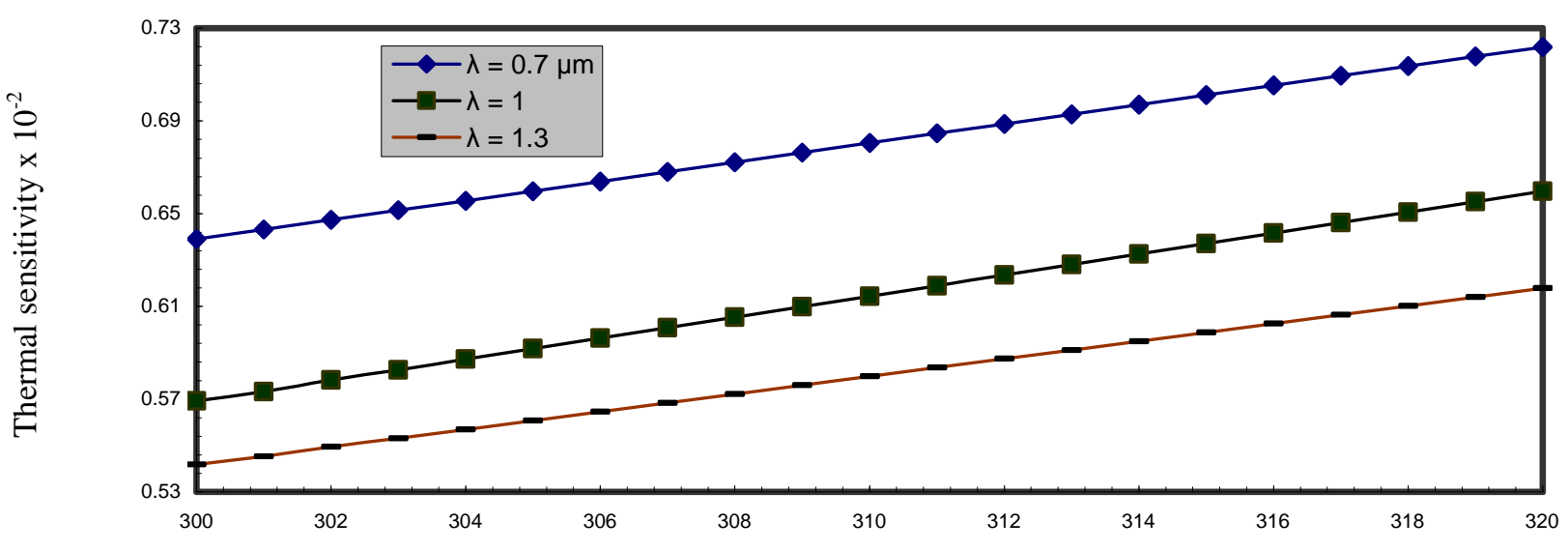

Material temperature $\mathrm{T}[\mathrm{K}]$

Fig. 10. Variation of thermal sensitivity versus temperature for $\mathrm{LiNbO}_{3}$ material

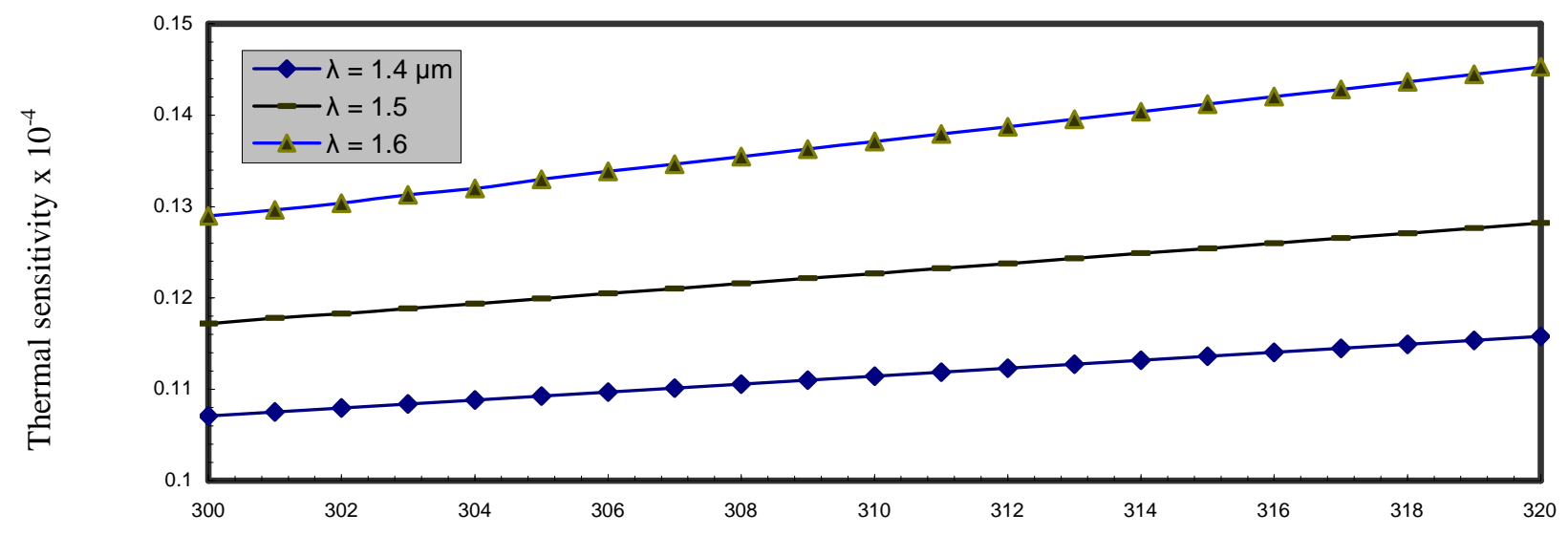

Material temperature $\mathrm{T}[\mathrm{K}]$

Fig. 11. Variation of thermal sensitivity versus temperature for Silica-doped material

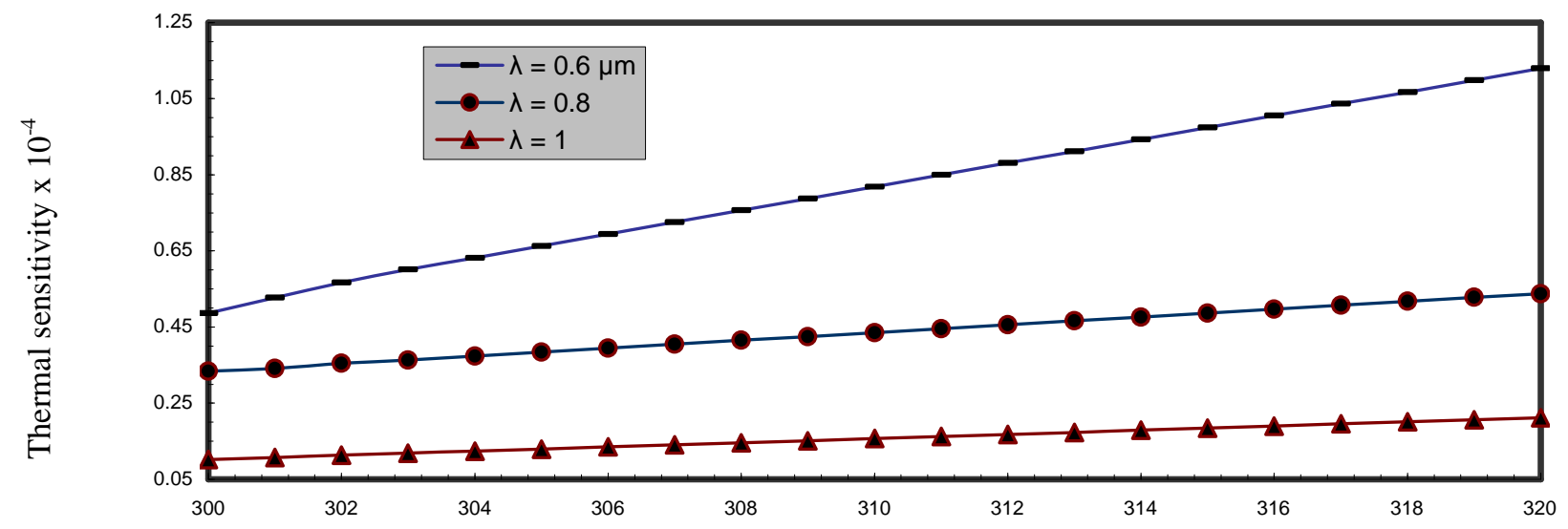

Material temperature $\mathrm{T}[\mathrm{K}]$

Fig. 12. Variation of thermal sensitivity versus temperature for PMMA material 